\title{
Leadership, innovation and engagement in quality improvement in the Ambulance Services Cardiovascular Quality Initiative: cross sectional study
}

\section{Nadya Essam ${ }^{1}$, Viet-Hai Phung, ${ }^{2}$, Zahid Asghar ${ }^{2}$, Anne Spaight ${ }^{1}$, A Niroshan Siriwardena ${ }^{1,2}$ ${ }^{1}$ East Midlands Ambulance Service NHS Trust, ${ }^{2}$ University of Lincoln}

\section{Introduction}

Clinical leadership and organisational culture are important contextual factors for successful Quality Improvement (QI) programmes. The relationship between these and with organisational performance is complex and poorly understood. We aimed to explore the relationship between leadership, culture of innovation, and clinical engagement in QI for organisations participating in a largescale national ambulance Quality Improvement Collaborative (QIC).

\section{Method}

We used a cross sectional survey design. An online questionnaire was distributed to 22,117 frontline ambulance staff across all 12 ambulance services in England. Scores (0-100\%) were derived for each key aspect: clinical leadership; culture of innovation; use of QI methods; and effectiveness of QI methods. Responses to an openended question were analysed and complemented the quantitative findings.

\section{Results}

There were $2,743(12 \%)$ responses from 11 of 12 participating ambulance services. Despite only a small proportion of responders (3\%) being directly involved with ASCQI, leadership behaviour was significantly higher for ASCQI members than for non-ASCQI members. Involvement in ASCQI was not significantly associated with responders' perceptions of the culture of innovation of their organisation, which was generally considered to be poor. ASCQI members were significantly more likely to use QI methods but overall uptake of QI methods was low. The use of QI methods was also significantly associated with leadership behaviour and service tenure.

\section{Supporting quotations}

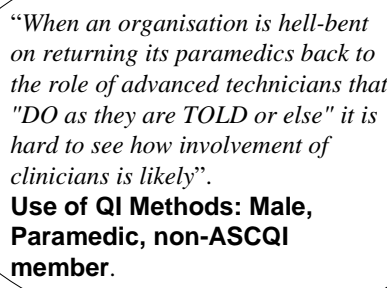

"When an organisation is hell-bent on returning its paramedics back to the role of advanced technicians that "DO as they are TOLD or else" it is hard to see how involvement of clinicians is likely".

Use of QI Methods: Male, Paramedic, non-ASCQI member.

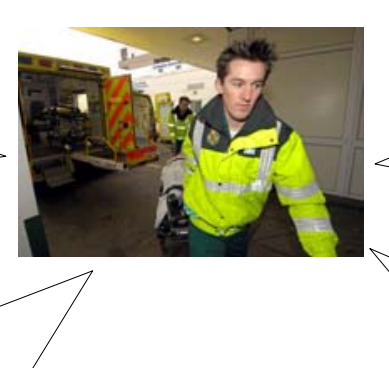

"Clinical staff in my Trust have frequently shown willingness to engage in quality improvement, research and audit”.

Use of QI methods: Male, Clinical / Paramedic

Team Leader, non-ASCQI member.
"Clinical staff in my trust have frequently shown willingness to engage in quality improvement, research and audit. I believe that if the leadership and resources are put in place then there would be widespread engagement and improved patient care as a result". Leadership for improvement: Male, Clinical/Paramedic Team Leader, non-ASCQI member.

\begin{abstract}
"Ideas and innovations are frequently ignored in place of target-based initiatives with little evidence base and no reward has ever been offered". Culture of Innovation: Male, Emergency Care Assistant (EMT1), nonASCQI member.
\end{abstract}

\section{Conclusion}

Although participants reported a lack of organisational culture of innovation, considered a prerequisite for QI, the collaborative achieved significant wide-scale improvements in prehospital care for myocardial infarction and stroke. We postulate that improvement was mediated through a 'QI subculture' developed from ASCQI's distributed leadership and network. Further research is needed to understand success factors for QI in different complex healthcare environments.

\section{Limitations}

This study involved a national survey across England involved 11 ambulance services. The response rate was low, limiting the generalisability of the study, but we were able to examine relationships between key variables and the free text responses enabled qualitative analysis and triangulation with the quantitative data. 